\title{
A classification system for the bicuspid aortic valve from 304 surgical specimens
}

Hans-H. Sievers, MD, and Claudia Schmidtke, MD, MBA

Earn CME credits at

http://cme.ctsnetjournals.org
Objective: In general, classification of a disease has proven to be advantageous for disease management. This may also be valid for the bicuspid aortic valve, because the term "bicuspid aortic valve" stands for a common congenital aortic valve malformation with heterogeneous morphologic phenotypes and function resulting in different treatment strategies. We attempted to establish a classification system based on a 5-year data collection of surgical specimens.

Methods: Between 1999 and 2003 a precise description of valve pathology was obtained from operative reports of 304 patients with a diseased bicuspid aortic valve. Several different characteristics of bicuspid aortic valves were tested to generate a pithy and easily applicable classification system.

Results: Three characteristics for a systematic classification were found appropriate: (1) number of raphes, (2) spatial position of cusps or raphes, and (3) functional status of the valve. The first characteristic was found to be the most significant and therefore termed "type." Three major types were identified: type 0 (no raphe), type 1 (one raphe), and type 2 (two raphes), followed by two supplementary characteristics, spatial position and function. These characteristics served to classify and codify the bicuspid aortic valves into three categories. Most frequently, a bicuspid aortic valve with one raphe was identified (type $1, \mathrm{n}=269$ ). This raphe was positioned between the left $(\mathrm{L})$ and right $(\mathrm{R})$ coronary sinuses in 216 (type 1, L/R) with a hemodynamic predominant stenosis (S) in 119 (type 1, L/R, S). Only 21 patients had a "purely" bicuspid aortic valve with no raphe (type 0).

Conclusions: A classification system for the bicuspid aortic valve with one major category ("type") and two supplementary categories is presented. This classification, even if used in the major category (type) alone, might be advantageous to better define bicuspid aortic valve disease, facilitate scientific communication, and improve treatment.

$\mathrm{B}$ icuspid aortic valve (BAV) is the most common congenital cardiac anomaly, with an estimated incidence of $0.9 \%$ to $2 \%$ in the general population. ${ }^{1,2}$ The term "BAV" includes different morphologic phenotypes ${ }^{3-6}$ presenting with different hemodynamic conditions. The "purely" BAV is composed of two cusps, morphologically and functionally. However, the most frequent form of a BAV consists of three developmental anlagen of cusps and commissures instead of two. Associated with a certain proportion of BAVs is a dilatation of the ascending aorta, especially in young patients, ${ }^{7}$ exposing these patients to an increased risk of comorbidity owing to aneurysm formation and dissection. In recent years, BAV has gained increasing interest in research and treatment. Owing to improved knowledge of aortic valve anatomy and function, more refined reconstructive techniques are applied to the different phenotypes of BAV. A systematic classification of BAV phenotypes seems advantageous to more precisely describe BAV, to better relate valve morphologic characteristics to surgical intervention, to gain more insight into 


$$
\begin{aligned}
& \text { Abbreviations and Acronyms } \\
& \begin{aligned}
\mathrm{B} & =\text { balanced insufficiency and stenosis } \\
\text { BAV } & =\text { bicuspid aortic valve } \\
\mathrm{I} & =\text { insufficiency } \\
\mathrm{L} & =\text { left } \\
\text { No } & =\text { no insufficiency and stenosis } \\
\mathrm{R} & =\text { right } \\
\mathrm{S} & =\text { predominant stenosis } \\
\mathrm{X} & =\text { nonclassifiable }
\end{aligned}
\end{aligned}
$$

the association of BAV with other disease of the ascending aorta, ${ }^{8}$ and to allow more appropriate comparison of the different reports on the BAV. The presented classification system is based on our surgical experience with BAVs over a 5-year period.

\section{Patients and Methods \\ Patients}

This is a single-center retrospective analysis of BAVs operated on from 1999 to 2003. In this time frame, 1206 aortic valve procedures were performed. A bicuspid or unicuspid aortic valve was found in 409 patients. Of these, 304 patients had a precise description of BAV valve pathology on operative reports, which are the basis of this study. The lack of precise information on 105 bicuspid and unicuspid valves was not valve related but due to missing awareness and intention to more precisely describe the morphologic characteristics useful for classification. The only information ascertainable from these operative reports was bicuspid or unicuspid valve, which was not enough for any kind of classification. This study was approved by the ethics committee.

Patient age at operation, gender, functional state of the patient (according to New York Heart Association classification), concomitant vascular diseases, and cardiac procedures, as well as the functional state of the aortic valve, were collected from the medical records.

\section{Functional State of the BAV and Indications for Operation}

The functional state of the BAV was determined before the operation by echocardiography and/or angiocardiography. The severity of aortic stenosis was assessed echocardiographically by the maximal pressure gradient $\left(=4 \times v^{2}([\mathrm{~m} / \mathrm{s}]\right.$, where $v$ is the peak systolic transvalvular velocity) and by the aortic valve area $\left(=\left[C S A_{L V O T} \times V T I_{L V O T}\right] / V T I_{A S}\right.$, where CSA is cross-sectional area, $L V O T$ is left ventricular outflow tract, $V T I$ is velocity time integral, and $A S$ is aortic stenosis). Aortic stenosis was also assessed angiographically by measuring the transvalvular pressure gradient directly and by the orifice area according to Gorlin and Gorlin.

An aortic stenosis was defined as severe if presenting with an orifice area of $1 \mathrm{~cm}^{2}$ or less or a mean pressure gradient of $50 \mathrm{~mm} \mathrm{Hg}$ or more. Aortic insufficiency was assessed by color flow Doppler techniques and graded by the ratio of jet height to left ventricular outflow tract height. Grade III aortic insufficiency (47\% to $64 \%$ of

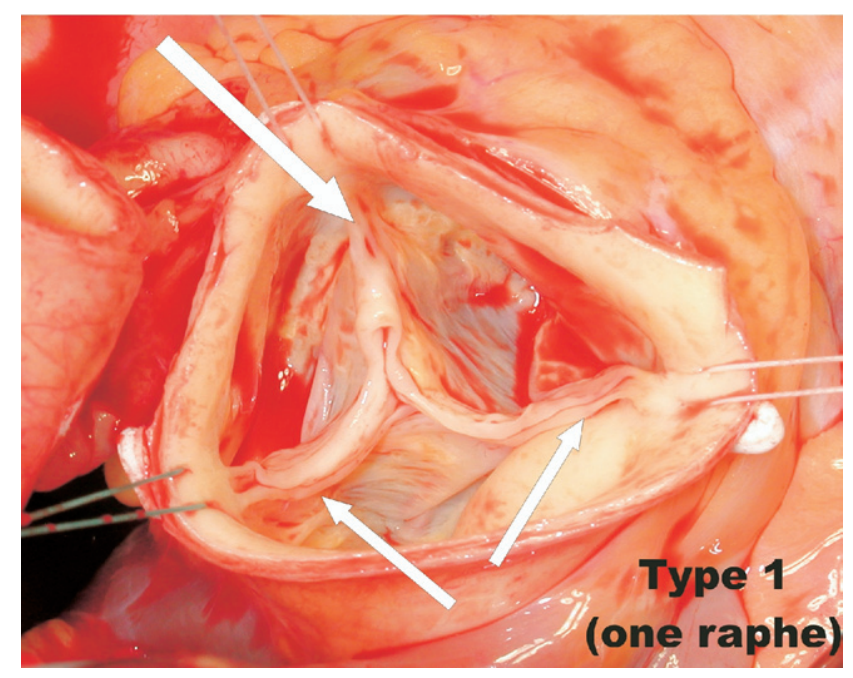

Figure 1. Intraoperative picture of a bicuspid aortic valve type 1, L/R, I (see text and Table 1 for explanation) with one completely developed noncoronary cusp, two completely developed commissures (small arrows), and one raphe between the underdeveloped left and right coronary cusps extending to the corresponding malformed commissure (large arrow) with hemodynamic signs of insufficiency due to prolapse of the conjoint cusps.

the aforementioned ratio) was considered an indication for operation in conjunction with the presence of symptoms and left ventricular size and function. Patients with severe aortic stenosis and symptoms or patients with an orifice area less than $0.7 \mathrm{~cm}^{2}$ were considered candidates for operative intervention. If aortic stenosis was combined with aortic insufficiency and both of moderate degree, indicating an operation, this valve condition was called a balanced lesion.

\section{Definition of BAVs}

In this study the term "BAV" stands for congenital bicuspid aortic valve disease comprising a spectrum of deformed aortic valves presenting on gross examination with two functional cusps forming a valve mechanism with less than three zones of parallel apposition between cusps. Thus, abnormal aortic valves with two raphes resulting in a restricted orifice area that extends from the periphery to the center were included in the BAV group (type 2, valve with two raphes) and not considered to be unicuspid, unicommissural, or monocuspid as described earlier for similar valve diseases. ${ }^{9-11}$ This is supported by the similarities in anatomy of BAV type 2 (valve with two raphes) to BAV type 1 (valve with one raphe).

Although the term "cusp" is a description more of a tip than an area and the term "leaflet" may be more appropriate, the term "cusp" is commonly used for the aortic valve and furthermore defines pathologic entities like the bicuspid and quadricuspid aortic valve. Therefore, we retain the term "cusp." The term "raphe" defines the conjoint or sometimes called "fused" area of the two underdeveloped cusps turning into a malformed commissure between both cusps (Figures 1 and 2). These raphes can be developed 


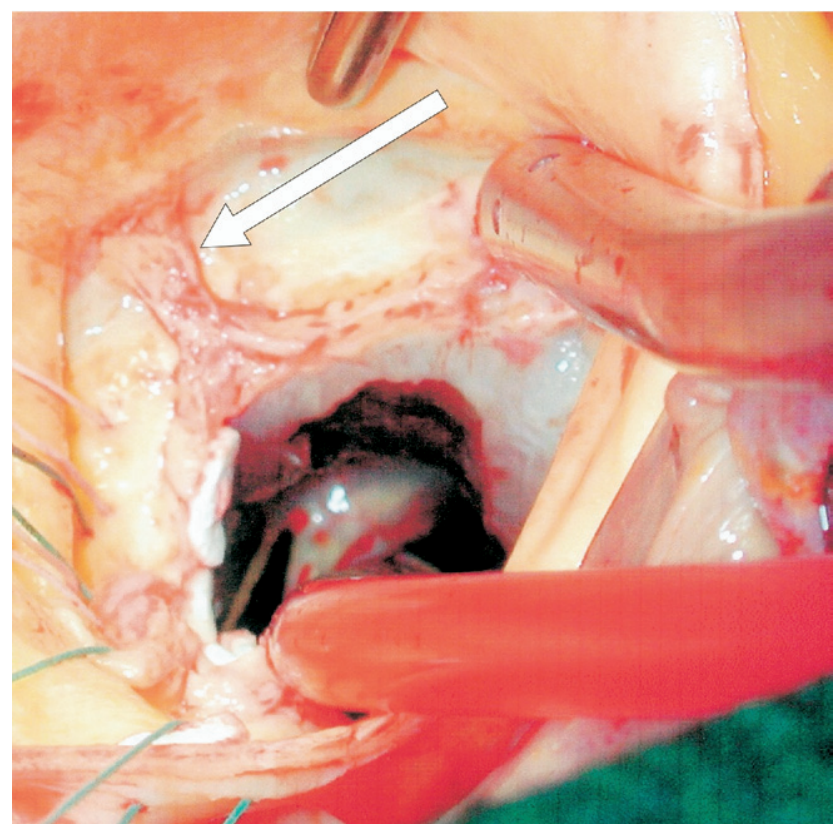

Figure 2. Situs after excision of a bicuspid aortic valve type 1, L/R, I (see text and Table 1 for explanation) showing the underdeveloped and obliterated commissure (arrow) between the left and right coronary cusps.

partially or totally. The characteristic appearance of a raphe is a ridge with many elastic fibers. ${ }^{12}$ The commissure of the aortic valve is the space between the two coronet-shaped, lateral, and parallel attachments of two adjacent cusps to the aortic wall normally not adhering to each other and is different from the zone of apposition between the free edges of adjacent cusps. In BAV, however, an obliteration of the commissural area is present (Figure 2).

Central to the pathology of a BAV is the malformation of a commissure (Figure 2) and the adjacent parts of the two corresponding cusps forming a raphe. There is a continuous spectrum of the BAV from completely missing one commissure, leading to two cusps, sinuses, and commissures only (Figures 3 and 4) to a more or less underdevelopment of one or two commissures and the adjacent cusps, which occurs in the majority of cases presenting with one or two raphes (Figures 1 and 5). In these cases the developmental anlagen of three cusps, commissures, and sinuses can be identified. Taken together, there are certain different morphologic characteristics of a BAV that allow, on gross examination, separation of the BAV from other aortic valve phenotypes (Figure 3), as already addressed in part by Osler $^{13}$ in 1886.

1. Two completely developed cusps, sinuses, and commissures, commonly called "purely" BAV.

2. Developmental anlagen of three cusps, commissures, and sinuses. However, one or two commissures are more or less malformed and obliterated, giving rise to a raphe, a fibrous ridge, which extends from the commissure to the free edge of the two underdeveloped, conjoint cusps.

2a. From the left ventricle, the commissural area presents as a indentation, not a space.

2b. The free edge of the conjoint cusps together is slightly larger than that of the opposite cusp.

2c. The circumferential distances among the three commissures are not equal.

\section{Description of the Classification System}

The classification system is based on three characteristics: the number of raphes, the spatial position of cusps or raphes, and the

\begin{tabular}{|c|c|c|c|c|c|c|}
\hline \multirow{2}{*}{\multicolumn{2}{|c|}{$\begin{array}{l}\text { Commonly used terms } \\
\text { Scheme of } \\
\text { morphological } \\
\text { appearance }\end{array}$}} & quadricuspid & tricuspid & \multicolumn{3}{|c|}{ bicuspid } \\
\hline & & & & & & \\
\hline $\begin{array}{l}\text { func- } \\
\text { tional } \\
\text { charac- } \\
\text { teristics }\end{array}$ & No of cusps & 4 & 3 & 2 & 2 & 2 \\
\hline \multirow{5}{*}{$\begin{array}{l}\text { morpho- } \\
\text { logical } \\
\text { charac- } \\
\text { teristics }\end{array}$} & No of raphes & 0 & 0 & 0 & 1 & 2 \\
\hline & & & & purely bicuspid* & \multicolumn{2}{|c|}{ potentially tricuspid* } \\
\hline & No of cusps & 4 & 3 & 2 & $\begin{array}{l}3 \text { anlagen, } \\
\text { ( } 2 \text { under- } \\
\text { and } 1 \text { fully } \\
\text { developed) }\end{array}$ & $\begin{array}{l}3 \text { anlagen, } \\
\text { ( } 2 \text { under- } \\
\text { and } 1 \text { fully } \\
\text { developed) }\end{array}$ \\
\hline & Size of cusps & non-equal & equal & equal & nonequal & non-equal \\
\hline & $\begin{array}{c}\text { No of } \\
\text { commissures }\end{array}$ & 4 & 3 & 2 & $\begin{array}{l}1 \text { under- } \\
\text { and } 2 \text { fully } \\
\text { developed }\end{array}$ & $\begin{array}{l}2 \text { under- } \\
\text { and } 1 \text { fully } \\
\text { developed }\end{array}$ \\
\hline
\end{tabular}

Figure 3. Schematic presentation of the developmental phenotypes of the aortic valve and typical characteristics. Prominent line in schematic drawings represents a raphe, which is the nonseparated or conjoint segment of two underdeveloped cusps extending into the commissural area. ${ }^{*}$ Angelini and associates. ${ }^{21}$ 

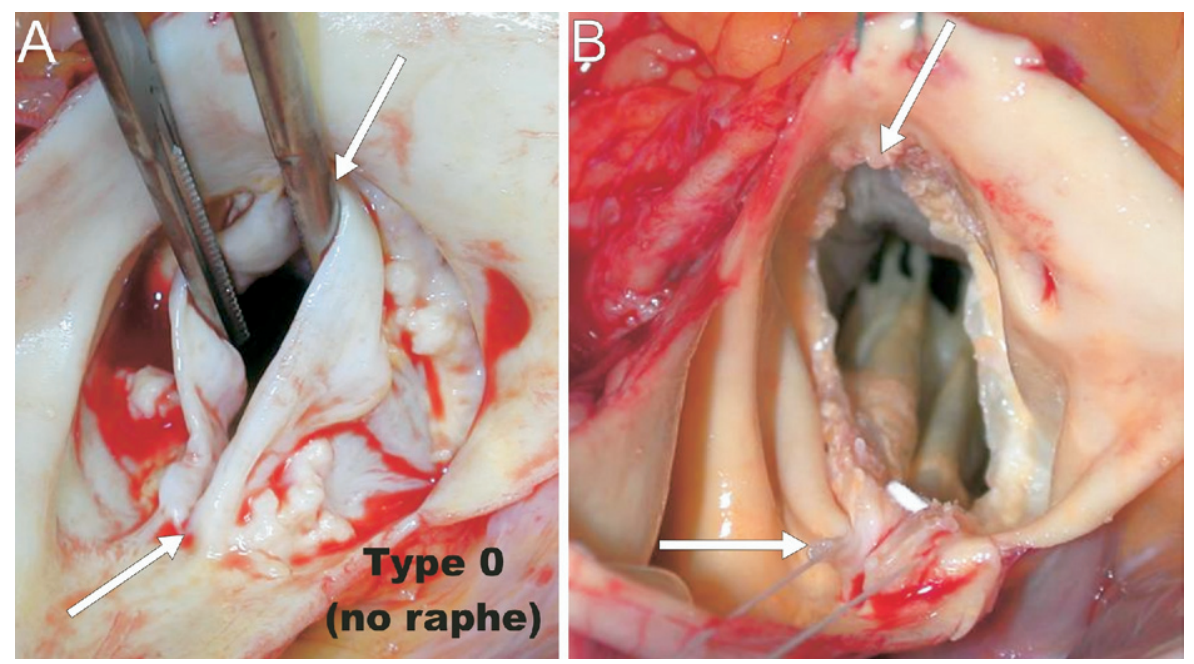

Figure 4. Intraoperative picture of a "purely" bicuspid aortic valve type 0 , lat, $\mathbf{S}$ (see text and Table 1 for explanation) with two equal-sized cusps, no raphe, lateral arrangement of the free edge of the cusps, and presenting predominantly with stenosis before (A) and after excision (B). Note that there are only two commissures (arrows).

functional status of the valve. BAVs were classified into three categories in accordance with the characteristics (Figure 3 and Table 1). The main category is of major importance, can stand on its own, and is therefore termed "type." The subcategories are supplementary. The main category represents the number of raphes, codifying the BAVs into three types: type 0 , valve with no raphe; type 1, valves with one raphe; and type 2, valves with two raphes.

The first subcategory relates to the spatial arrangements of the free edge of cusps in type 0 (valve with no raphe) and the raphes in types 1 and 2 (valve with one and valve with two raphes) as detailed in Table 1. For type 0 (valve with no raphe), the orienta-

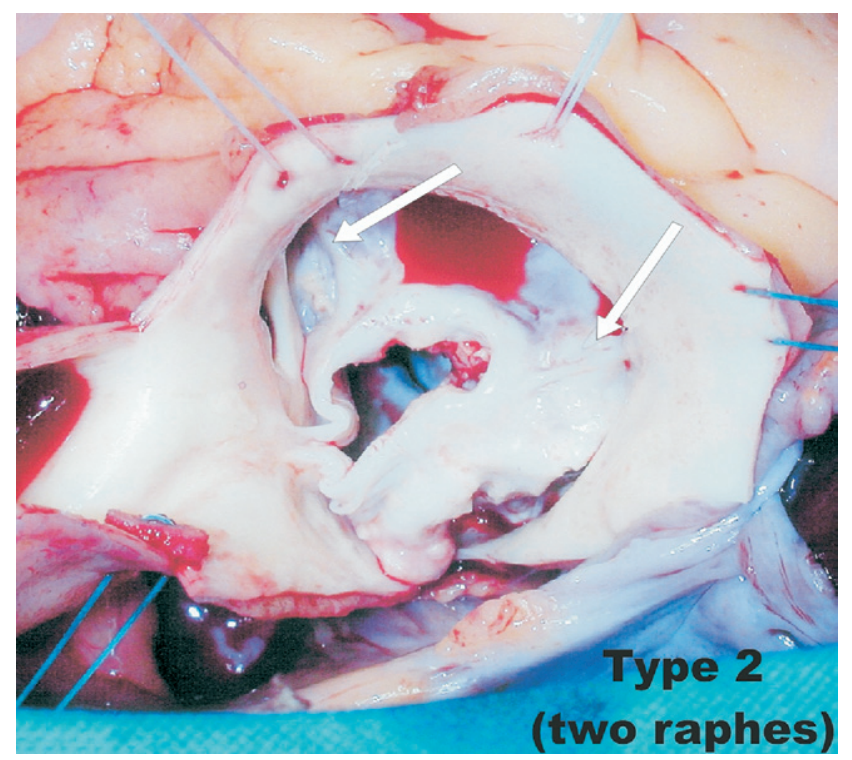

Figure 5. Intraoperative picture of a bicuspid aortic valve type 2, L/R-R/N, S (see text and Table 1 for explanation) with two raphes (arrows) but developmental anlagen of three cusps with a high degree of stenosis. tion of the free edge of the cusps was found to be either anteroposterior or lateral. For types 1 and 2 (valve with one raphe and valve with two raphes), the orientation of the raphes in relation to the sinuses defines this subcategory; for example, BAV type 1, $\mathrm{L} / \mathrm{R}$, means BAV type 1 (valve with one raphe) with the raphe positioned between the left (L) and right (R) coronary sinuses.

The second subcategory is determined by the functional status of the valve: predominant insufficiency (I), predominant stenosis (S), balanced insufficiency and stenosis (B), or no insufficiency and stenosis (No).

A missing or nonclassifiable subcategory is presented by an "X."

Thus, the classification system of a BAV may be presented in three blocks - type, spatial position, and valvular function- using the aforementioned codifications.

\section{Statistical Analysis}

Categorical data are given as total numbers and relative frequencies. Continuous data are given as mean \pm standard deviation. Characteristics for patients of different groups were compared by the Fisher exact test for categorical variables and the $U$ test and Kruskal-Wallis test for continuous variables. Statistical analyses were performed with statistical software SAS (SAS 8.2; SAS Inc, Cary, NC).

\section{Results}

\section{Patient Characteristics and Operations}

Mean age of $237(77.9 \%)$ male and 67 (22.1\%) female patients was $53 \pm 15.4$ years (range 14.6-82.5 years) (Figure 6). Patients with BAV type 2 (valve with two raphes) were significantly younger at operation $(P=.007,40$ years for type 2 versus 53 years for type 1 [valve with one raphe] and 51 years for type 0 [valve with no raphe]). The proportion of male to female subjects was 3.5 to 1 .

Preoperatively, most patients were in a clinical condition corresponding to New York Heart Association class II (mean value $2.1 \pm 0.84$ ). Body surface area was $1.96 \pm$ 
TABLE 1. Schematic presentation (as viewed from the surgeon's position with the left coronary sinus on the left side) of the classification system of BAVs with one main and two subcategories, including the number of specimens (percent in parenthesis)

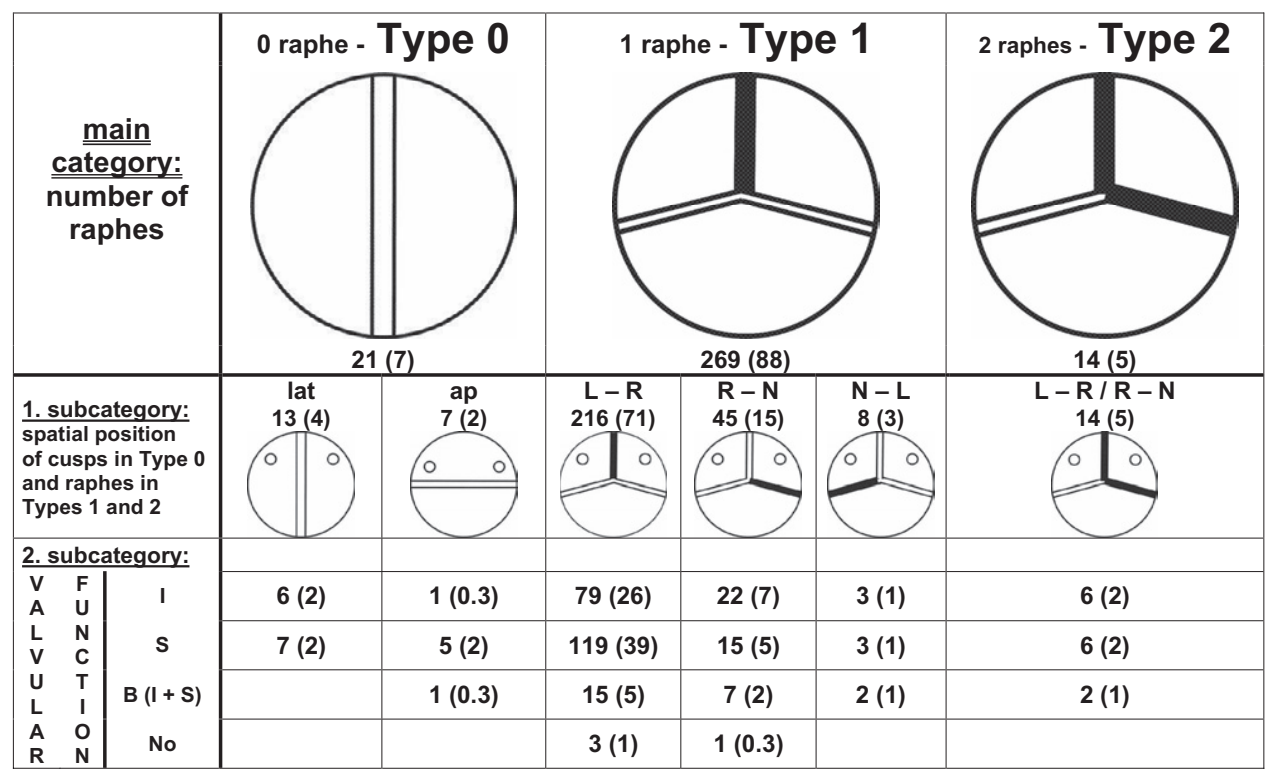

Prominent lines in schematic drawings represent a raphe. The codification for the main category is Type (Type 0 for value with no raphe; Type 1 for valve with one raphe; Type 2 for valve with two raphes); for the first subcategory: ap, lat, L-R, R-N, N-L, and L-R/R-N; and for the second category: I, S, B, and No. Thus, the classification system may be presented as a codification in three blocks: Type, spatial position, and valvular function (eg, BAV Type 1, L-R, $S$, meaning a bicuspid aortic valve with one raphe between the left and right coronary sinus, and with valvular stenosis, which was the most frequent specimen). One specimen of a BAV Type 0 (valve with no raphe) could not be categorized in the first subcategory, resulting in the codification BAV Type $0, \mathrm{X}, \mathrm{S}$. ap, anterior-posterior; lat, lateral; $L$, left coronary sinus; $R$, right coronary sinus; $N$, non-coronary sinus; $I$, insufficiency; $S$, stenosis; $B$, balanced valvular lesion; No, normal function; $B A V$, bicuspid aortic valve; $X$, not ascertainable.

$0.22 \mathrm{~m}^{2}$. Concomitant diseases were coronary artery disease in $86(28.3 \%)$, hypertension in $167(54.9 \%)$, hyperlipidemia in $111(36.5 \%)$, diabetes in $31(10.2 \%)$, Marfan syndrome in $2(0.7 \%)$, and history of cancer in $17(5.6 \%)$.

The underlying aortic valve disease was an insufficiency in $117(38.5 \%)$, stenosis in $156(51.3 \%)$, balanced combined

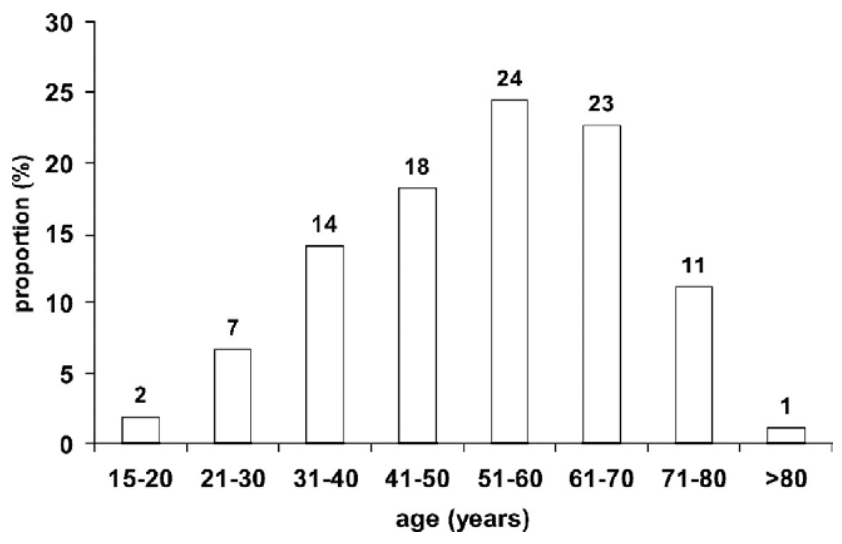

Figure 6. Age distribution of the 304 patients with a bicuspid aortic valve. valve disease in $27(8.9 \%)$, and active endocarditis in 15 (4.9\%) patients; 4 of these were without valve dysfunction but had large vegetations.

Aortic aneurysms (diameter $>5 \mathrm{~cm}$ ) were present in 90 (29.6\%) patients, with involvement of the aortic root in 18 (5.9\%), ascending aorta in $88(28.9 \%)$, aortic arch in 2 $(0.7 \%)$, and descending aorta in $1(0.3 \%)$. A significantly higher proportion of aneurysms of the ascending aorta was present in BAV type 2 (valve with two raphes; $P=.022$; Figure 7).

Most patients had a Ross procedure $(\mathrm{n}=111,36.5 \%), 96$ $(31.6 \%)$ a bioprosthesis, and $72(23.7 \%)$ a mechanical prosthesis (as a conduit/Bentall procedure in 17, 5.6\%). Reconstruction of the valve was performed in $17(5.6 \%)$, reimplantation technique according to David and Feindel ${ }^{14}$ in 5 (1.6\%), and remodeling technique according to Yacoub and associates ${ }^{15}$ in $3(1.0 \%)$.

There was no difference in coronary artery disease between types of BAVs (33\% for type 0 [valve with no raphe], $29 \%$ for type 1 [valve with one raphe], and $14 \%$ for type 2 [valve with two raphes]).

Concomitant procedures were performed as follows: coronary artery bypass grafting in $43(14.1 \%)$, maze procedure 


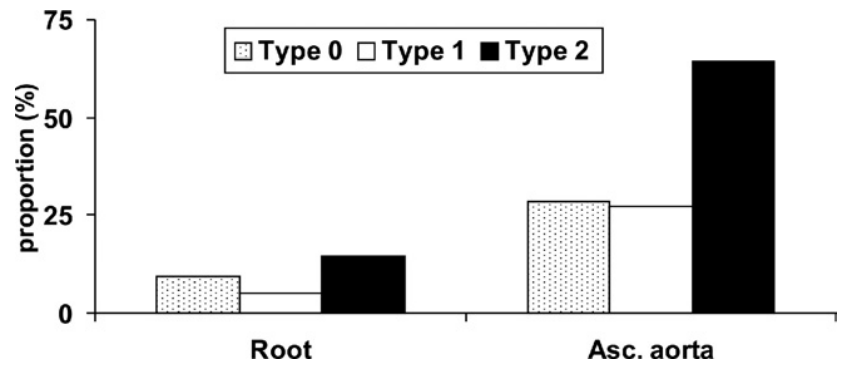

Figure 7. Proportion of an aneurysm of the aortic root or ascending aorta in relation to the type of bicuspid aortic valve. A bicuspid aortic valve type 2 (valve with two raphes) was associated with a significantly $(P=.022)$ higher proportion of aneurysms.

in $4(1.3 \%)$, left ventricular myectomy in $7(2.3 \%)$, mitral valve reconstruction in $16(5.3 \%)$, closure of a patent foramen ovale in $3(1.0 \%)$, and closure of a ventricular septum defect in $1(0.3 \%)$.

\section{Classification of Surgical Specimens}

The results are summarized in detail in Table 1. There were $21(7 \%)$ patients with BAV Type 0 (valve with no raphe). One of these specimens with predominant valvular stenosis could not be categorized into the first subcategory owing to lack of specification of the orientation of cusps in the operative report, resulting in the codification type $0, \mathrm{X}, \mathrm{S}$. Most patients $(\mathrm{n}=269,88 \%)$ had BAV type 1 (valve with one raphe). Within this group the predominant finding was type $1, \mathrm{~L} / \mathrm{R}, \mathrm{S}(\mathrm{n}=119,39 \%)$, which means a BAV with one raphe between the left and right coronary sinuses and with valvular stenosis. There were 14 patients with BAV type 2 (valve with two raphes).

\section{Discussion}

The BAV, appearing on gross examination as an aortic valve with two cusps, has clinical relevance ${ }^{16-18}$ and has gained increasing interest in the medical community from a pathogenetic and especially surgical point of view. ${ }^{19,20}$ The BAV represents a certain segment of the developmental spectrum of aortic valve phenotypic continuity. Developmentally, the BAV is not simply the fusion or nonseparation of two cusps but a more complex process involving the cusps, sinuses, commissures, position of the coronary orifices, and possibly the texture of the wall of the ascending aorta. BAVs are not equal among each other in morphologic, positional, and functional aspects, which is of significance for treatment. We found the number of raphes to be the most practical and readily recognizable distinctive characteristic of BAVs, representing the main category of this classification, called "type." Typically, no raphe is present in the so-called "purely" BAV with two completely devel- oped cusps and commissures (type 0), which is, however, the case in the minority of patients (7\% in this series); this is comparable with the results of Angelini and colleagues. ${ }^{21}$ In this configuration, valve implantation in general, and especially the Ross procedure, is more challenging, and a circular, not scalloped, proximal suture line seems preferable. In addition, the vis-à-vis orientation of the coronary ostia requires special consideration when arranging the three new commissures of the autograft. In roots with this kind of unfavorable anatomy, it is sometimes advisable not to perform a Ross procedure.

In BAV type 1 (valve with one raphe), there are two smaller malformed cusps bearing the conjoint part or raphe and one larger cusp, most frequently the noncoronary. The commissure between both underdeveloped cusps is also malformed. This is, however, of no major surgical concern because the surgical annulus for implantation of prostheses and also for the Ross procedure is largely maintained (Figure 2) or even flattened to a more ringlike configuration in relation to the extent of malformation of this commissural area. The surgical annulus is composed of the proximal part of the semilunar attachments of the cusps to the wall, extending from the nadir of the sinuses to the point where this line approaches that of the adjacent cusp. ${ }^{22}$ The larger noncoronary annulus and sinus can easily be matched by a plication suture at the nadir and/or the sinus during closure of the aortotomy. Even in BAV type 2 with two raphes, three sinuses are identifiable, facilitating the pulmonary autograft procedure or conventional valve replacement.

In patients with an insufficient BAV type 1 (valve with one raphe), usually the two conjoint leaflets with the raphe are prolapsing, giving the appearance that there is some cusp tissue missing at the area of the raphe. This can be replaced by prosthetic material or pericardial tissue, but preferably the raphe is plicated or resected and thus the cusps are raised up to the coaptation area of the nonprolapsing cusp. This procedure, however, produces inevitably some degree of stenosis, which seems not to be of clinical significance even at exercise. ${ }^{23}$ Nevertheless, the deficiency of cusp tissue may require other surgical techniques, such as annuloplasty or cusp extension, to increase coaptation area or elevation of commissures.

In BAVs type 0 (valve with no raphe), plication of the prolapsing cusp also restores coaptation area. Long-term results of reconstruction of insufficient BAV type 1 (valve with one raphe) and type 0 (valve with no raphe) are likely different partly as a result of different morphologic characteristics.

The first subcategory is related to the position of the raphes. This is of minor direct surgical impact but may have some pathophysiologic relevance. In this respect, it is of interest that BAVs type 2 (valve with two raphes), L/R-R/N were associated with the highest incidence of aneurysms of 


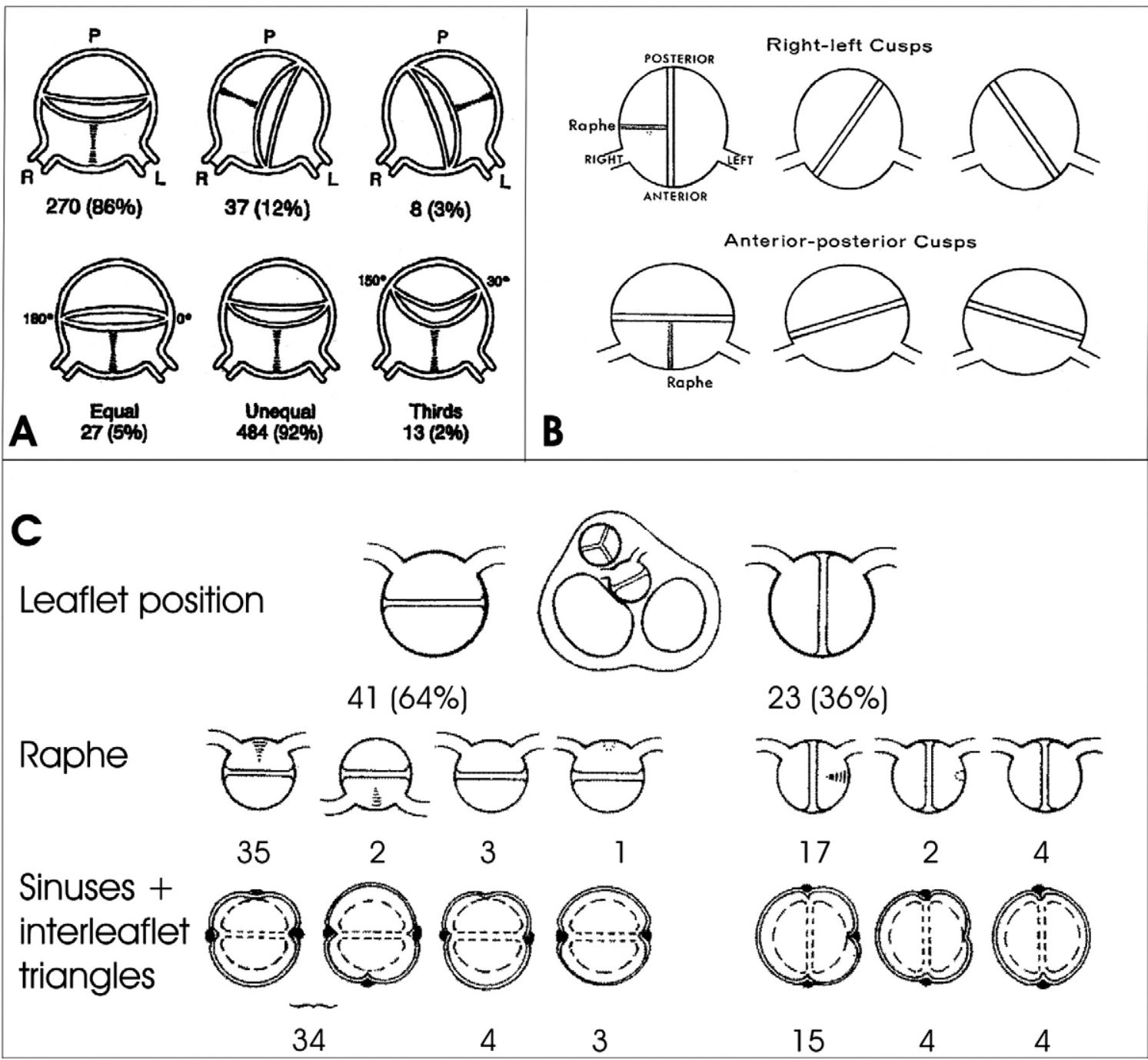

Figure 8. Schemes of bicuspid aortic valve classifications of different authors: A, Sabet and associates ${ }^{4}$; B, Roberts and associates ${ }^{12}$; C, Angelini and associates. ${ }^{21}$ The position of the raphe and cusps, the relative sizes of cusps, as well as the number of sinuses and interleaflet triangles as described from the left ventricular outflow were main but not uniform determinants for classifying bicuspid aortic valves.

the ascending aorta (Figure 7). The flow through this reduced opening area is directed to the convexity of the ascending aorta, where typically the aneurysms are located. Whether this increased local wall stress acts as a trigger for aneurysm formation is questionable. Probably these typical dilatations of the ascending aorta are related more to BAV intrinsic aortic disease than to hemodynamics.

The second subcategory provides information on the functional status of the valve, which is not only of diagnostic interest but also may have some prognostic information on ventricular performance, timing of operation, and operative technique.

With this classification system, it was possible to group all BAV phenotypes at least by the main category.
For other studies on BAV morphology it was also possible to apply this classification system to the main category. ${ }^{3,4,6,21}$ Other authors ${ }^{4,5,9,21}$ principally found similar results concerning the distribution of purely BAVs and those with one raphe, as well as the position of the raphes. They also used the position of both the cusps and the raphes, the number of sinuses, the interleaflet triangles from below, and the cusp sizes for BAV description (Figure 8). These classifications, however, are not uniform, not widely used, and are not condensed in a concise, easily applicable, graduated classification and codification including morphology (number of raphes), spatial position of cusps or raphes, and valvular function, 
nor were abnormal aortic valves with two raphes included in the BAV group. From a surgical point of view especially, the number and position of raphes is easily assessable and applicable for classifying and codifying BAVs in the vast majority of patients.

Nevertheless, this classification system has some limitations. Valve calcification may render the classification difficult, especially if a congenital tricuspid valve becomes calcified, leading to acquired fusion of the cusps. However, after excision of the calcified leaflets, the developmental status of the commissures can be identified in most cases. An obliterated commissure is typical for BAV (Figure 2). This also holds true for a BAV with two raphes (type 2) and corresponding underdeveloped commissures. Furthermore, the assessment of the distance among the three commissures, which is more or less equal in tricuspid valves, but not equal in most BAVs, may help for classification. ${ }^{12}$ In addition, the coronary artery anatomy with a higher incidence of left main dominance ${ }^{7}$ might help the identification of a BAV in these cases. On the other hand, if there is some kind of a raphe in a "purely" BAV with two cusps, which is the proposed type 0 (valve with no raphe), there is no category in the described system for classifying this valve. However, these cases are extremely uncommon and may be described separately.

It is imaginable that additional subcategories could even more precisely specify the valve, for example, size of cusps, ${ }^{4}$ the concomitant existence of an ascending aortic aneurysm, extension and size of raphes, microscopic details, genetic markers, heritability signs,${ }^{24}$ and annulus dilatation, just to mention some. However, these additional items would make the classification system more complex and probably less practical.

We believe that the proposed classification, with the main category termed "type" alone or preferably together with the two subcategories, would yield advantages to more precisely and comparably describing the BAV as a further step for improved management of this entity.

We thank Anja Kumme for the excellent help in the data collection.

\section{References}

1. Ward C. Clinical significance of the bicuspid aortic valve. Heart. 2000;83:81-5.

2. Gray GW, Salisbury DA, Gulino AM. Echocardiographic and color flow Doppler findings in military pilot applicants. Aviat Space Environ Med. 1995;66:32-4.
3. Roberts WC, Ko JM. Frequency by decades of unicuspid, bicuspid and tricuspid aortic valves in adults having isolated aortic valve replacement for aortic stenosis, with or without associated aortic regurgitation. Circulation. 2005;111:920-5.

4. Sabet HY, Edwards WD, Tazelaar HD, Daly RC. Congenitally bicuspid aortic valves: a surgical pathology study of 542 cases (1991 through 1996) and a literature review of 2715 additional cases. Mayo Clin Proc. 1999;74:14-26.

5. Fernandes SM, Sanders SP, Khairy P, Jenkins KJ, Gauvreau K, Lang $\mathrm{P}$, et al. Morphology of bicuspid aortic valve in children and adolescents. J Am Coll Cardiol. 2004;44:1648-51.

6. Lerer PK, Edwards WD. Coronary arterial anatomy in bicuspid aortic valve necropsy study of 100 hearts. Br Heart J. 1981;45:142-7.

7. Nistri S, Sorbo MD, Marin M, Palisi M, Scognamiglio R, Thiene G. Aortic root dilatation in young men with normally functioning bicuspid aortic valves. Heart. 1999;82:19-22.

8. Ferencik M, Pape LA. Changes in size of ascending aorta and aortic valve function with time in patients with congenitally bicuspid aortic valves. Am J Cardiol. 2003;92:43-6.

9. Edwards JE. Pathology of left ventricular outflow tract obstruction. Circulation. 1965;31:586-99.

10. McKay R, Smith A, Leung MP, Arnold R, Anderson RH. Morphology of the ventriculoaortic junction in critical aortic stenosis. $J$ Thorac Cardiovasc Surg. 1992;104:434-42.

11. Falcone MW, Roberts WC, Morrow AG, Perloff JK. Congenital aortic stenosis resulting from a unicommissural valve. Circulation. 1971;44: $272-80$.

12. Roberts WC. The congenital bicuspid aortic valve. Am J Cardiol. 1970;26:72-83.

13. Osler W. The bicuspid condition of the aortic valves. Trans Assoc Am Physicians. 1886;2:185-92.

14. David TE, Feindel M. An aortic valve-sparing operation for patients with aortic incompetence and aneurysm of the ascending aorta. $J$ Thorac Cardiovasc Surg. 1992;103:617-22.

15. Yacoub MH, Gehle P, Chandrasekaran V, Birks EJ, Child A, RadleySmith R. Late results of a valve-preserving operation in patients with aneurysms of the ascending aorta and root. J Thorac Cardiovasc Surg. 1998;115:1080-90.

16. Fedak PWM, Verma S, David TE, Leask R, Weisel RD, Butany J. Clinical and pathophysiological implications of a bicuspid aortic valve. Circulation. 2002;106:900-4.

17. Edwards WD, Leaf DS, Edwards JE. Dissecting aortic aneurysm associated with congenital bicuspid aortic valve. Circulation. 1978;57: $1022-5$.

18. Lewin MB, Otto CM. The bicuspid aortic valve. Adverse outcomes from infancy to old age. Circulation. 2005;111:832-4.

19. Kuralay E, Demirkilic U, Özal E, Öz BS, Cingoz F, Günay C, et al. Surgical approach to ascending aorta in bicuspid aortic valve. J Card Surg. 2003;18:173-80.

20. Nash PJ, Vitvitsky E, Li J, Cosgrove DM, Pettersson G, Grimm RA. Feasibility of valve repair for regurgitant bicuspid aortic valves-an echocardiographic study. Ann Thorac Surg. 2005;79:1473-9.

21. Angelini A, Ho SY, Anderson RH, Devine WA, Zuberbuhler JR, Becker AE, et al. The morphology of the normal aortic valve as compared with the aortic valve having two leaflets. J Thorac Cardiovasc Surg. 1989;98:362-7.

22. Sievers HH. Prosthetic aortic valve replacement. J Thorac Cardiovasc Surg. 2005;129:961-5.

23. Schmidtke C, Poppe D, Dahmen G, Sievers HH. Echocardiographic and hemodynamic characteristics of reconstructed bicuspid aortic valves at rest and exercise. Z Kardiol. 2005;94:437-44.

24. Cripe L, Andelfinger G, Martin LF, Shooner K, Woodrow D. Bicuspid aortic valve is heritable. $J$ Am Coll Cardiol. 2004;44:138-43. 